\title{
Multiple levels in intermediate band solar cells
}

\author{
Antonio Luque, ${ }^{1}$ Pablo G. Linares, ${ }^{1, a)}$ Elisa Antolín, ${ }^{1}$ Enrique Cánovas, ${ }^{1}$ Corrie D. Farmer, ${ }^{2}$ \\ Colin R. Stanley, and Antonio Martí ${ }^{2}$ \\ ${ }^{1}$ Instituto de Energía Solar. Universialad Politécnica de Madrid, 28040 Madrid, Spain \\ ${ }^{2}$ Department of Electronics and Electrical Engineering, University of Glasgow. Glasgow G12 8QQ. \\ United Kingdom
}

\begin{abstract}
The presence of multiple energy levels in the intermediate band solar cell (IBSC) is studied by detailed balance calculations under ideal conditions. Multiple levels are found experimentally in IBSCs made with quantum dots (QDs) which act to reduce the limiting efficiency determined from detailed balance calculations. $J_{\mathrm{L}}-V_{\mathrm{OC}}$ measurements up to 1000 suns on IBSCs are presented together with their fitting to modified detailed balance calculations. It is found that the introduction of the QDs degrades the performance of the host cell but the sub-bandgap cell operates close to ideality.
\end{abstract}

The intermediate band solar cell (IBSC) was proposed ${ }^{1}$ as a way to increase the conversion efficiency of solar cells (SCs). A very high efficiency, with a detailed balance limit of about $63 \%$ versus $41 \%$ for a single junction SC, was calculated. Subsequently, prototype cells have been fabricated by several groups using GaAs quantum dots (QDs) in a GaAs matrix, ${ }^{2-4}$ although their efficiencies do not yet approach those of good single junction GaAs cells. A number of reasons for this degraded performance have been discussed. ${ }^{5}$ Several confined levels above the fundamental one are found experimentally ${ }^{6}$ and also by quantum calculations, ${ }^{7}$ which we refer to generically as the intermediate band (IB). The purpose of this letter is to investigate the effect of these levels on the IBSC performance. We will compare measured results with idealized values derived from detailed balance calculations in an effort to obtain further information on the issues that hamper the achievement of the high efficiencies predicted by theory.

In a single level IBSC, three absorption bands are capable of producing electron-hole pair generation; the valence band (VB) $\rightarrow$ conduction band (CB) transition (the only one in single gap cells, i.e., cells unmodified with QDs), the $\mathrm{VB} \rightarrow \mathrm{IB}$ transition and the $\mathrm{IB} \rightarrow \mathrm{CB}$ transition. The three bands (the IB being multilevel) are sketched in Fig. I(a), where $E_{\mathrm{v}}(\equiv 0)$ represents the top of the $\mathrm{VB}, E_{\mathrm{c}}$ the bottom of the $\mathrm{CB}$ (main band gap), and $E_{\mathrm{ti}}(i=1,2,3$, and 4 ) the position of each of the QD electron confined levels above $E_{\mathrm{y}}$. Although many QD confined hole levels are present within the VB offset, they are very close to each other and thus regarded as the uppermost of the VB.

In the single level model, the IB is located at a distance $E_{\mathrm{t}}$ from $E_{\mathrm{v}}$ and therefore, any photon with energy $E$ in the interval $\left(E_{\mathrm{c}}-E_{\mathrm{t}}\right)<E<E_{\mathrm{t}}$ [for the case of $\left(E_{\mathrm{c}}-E_{\mathrm{t}}\right)<E_{\mathrm{t}}$ ] is assumed to produce an IB $\rightarrow \mathrm{CB}$ transition. Furthermore, any photon with energy $E_{\mathrm{t}}<E<E_{\mathrm{c}}$ is assumed to produce only $\mathrm{VB} \rightarrow \mathrm{IB}$ transitions (although energetically, it might also produce a IB $\rightarrow C B$ transition, this is assumed to be forbidden in our analysis). Any photon with energy $E_{\mathrm{c}}<E$ is assumed to produce a VB $\rightarrow$ CB transition (although energetically, it might also produce $a \mathrm{IB} \rightarrow \mathrm{CB}$ or a $\mathrm{VB} \rightarrow \mathrm{IB}$

a'Electronic mail: p.garcia-linares@ies-def.upm.es. transition, again we assume the probability of this to be zero). In the ideal behavior, all the photons in these ranges are absorbed and produce the desired electron transitions.

In 2004, we published a generalized equivalent circuit for the IBSC. ${ }^{2}$ One of the main components of this circuit is the host subcell. It consists of a photocurrent generator in parallel with a diode representing $\mathrm{VB} \rightarrow \mathrm{CB}$ pumping and recombination, respectively. In parallel with this host subcell there is a series combination of two further subcells, representing pumping and recombination for the $\mathrm{VB} \rightarrow \mathrm{IB}$ and the $\mathrm{IB} \rightarrow \mathrm{CB}$ transitions. Each generation term is given by;

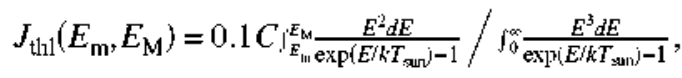

which represents all the photons available from the sun, characterized by a black-body at a temperature $T_{\text {sun }}=6000 \mathrm{~K}$ (a rough approximation used in this type of calculation which neglects details of the real solar spectrum) in a range $\left(E_{\mathrm{m}}, E_{\mathrm{M}}\right)$ in electronvolts and restricted in solid angle so as to produce a power flux of $0.1 C \mathrm{~W} \mathrm{~cm}^{-2}$ (where $C$ is the concentration).

The recombination terms, which are the detailed-balance counterpart of the generation terms, are given approximately by the van Roosbroeck-Shockley formula ${ }^{8}$

$$
J_{\mathrm{thlr}}\left(E_{\mathrm{m}}, E_{\mathrm{M}}\right)=\frac{2 \pi}{h^{2} c^{3}} \int_{E_{\mathrm{n} t}}^{E_{\mathrm{M}}} E^{2} \exp \left[-\left(E-F_{\mathrm{t}}\right) / k T\right] d E,
$$

where $F_{\mathrm{t}}$ represents the IB quasi-Fermi level (QFL) (located at the level $E_{t}$ ) and $T$ is the cell temperature. The QFL is
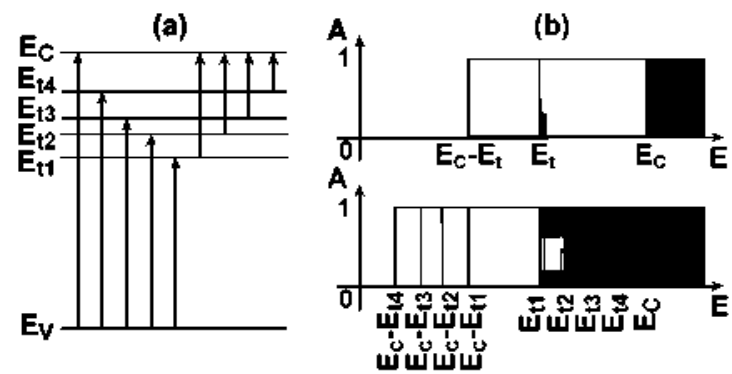

FIG. 1. (a) Schematic of the transitions between the IB levels and the C.B and VB. (b) Atsorption bands for the single-level (upper diagran) and the four-level (lower diagram) IBSC cases. 


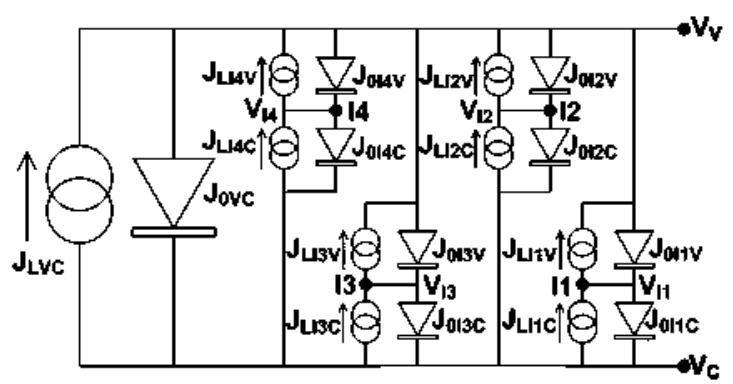

FIG. 2. Equivalent circuit for a four-level IBSC. The voltages correspond to the $Q F L$ at the different energy levels or bands. with a change of sign $\left(E_{\mathrm{F}}=-e V\right)$.

constant throughout the whole cell volume as a consequence of the infinite mobility assumed in ideal cases.

The multiple level case is a generalization of the single level case. As represented in Fig. I(a), transitions are now permitted from the VB to each $E_{t i}$ level and from each of these levels to the CB. We assume that every photon is absorbed in the most favorable manner, i.e., by the interband transition with the largest threshold energy. The absorption intervals are now represented in Fig. 1(b). The generation and recombination terms are given by Eqs. (1) and (2) using the appropriate intervals, and the resulting equivalent circuit is shown in Fig. 2.

Several QD-IBSCs have been manufactured and characterized. The cell labeled A 1681 has $\mathrm{QD}$ transition energies at $1.052,1.137,1.175$, and $1.225 \mathrm{eV}$ as well as a clear wetting layer (WL) signature at $1.35 \mathrm{eV}$. This WL is a quantum well generated by the Stranski-Krastanov QD growth mode, and its value also accounts for the effect of the previously mentioned VB offset and other factors that reduce the main band gap width. ${ }^{5,6}$ The detailed balance $J-V$ curves are presented in Fig. 3.

If we keep our analysis within the detailed balance realm, no transitions can occur between the several QD levels because the associated energy interval for integration is zero (assuming singular energy levels for simplicity). However, electron transfer among the different levels not involving a photon has a strong probability. The limit is the case in which all the levels have a single QFL. In the equivalent circuit of Fig. 2, this would correspond to a wire connection between the points labeled as I1, I2, I3, and I4. The result corresponding to this case (single QFL IBSC) is represented

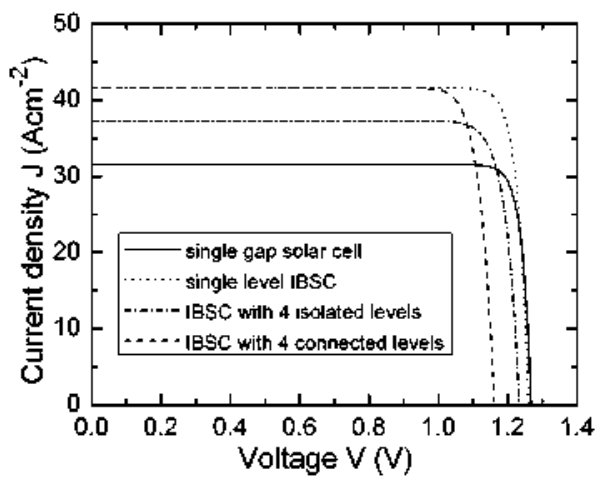

FIG. 3. Detailed balance $J-V$ calculations for a single junction cell at $C=1000$ suns with an equivalent band gap of $1.35 \mathrm{eV}$ for the following cases: IBSC with one level at $1.052 \mathrm{eV}$; IBSC with four isolated levels (and four QFLs) with energies at $1.052,1.137,1.175$, and $1.225 \mathrm{eV}$; single QFL IBSC A single gap SC $J-V$ calculation without QD levels is also presented.

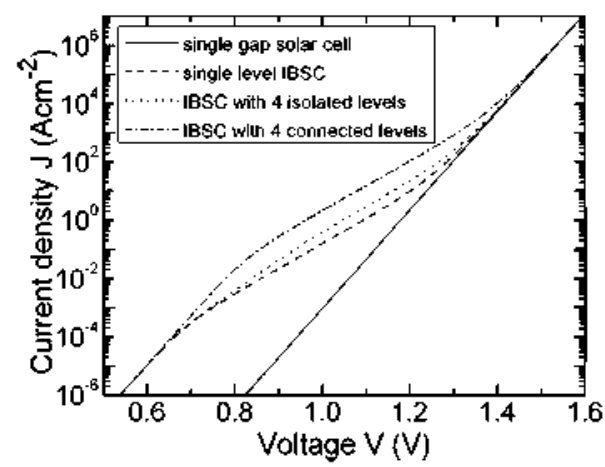

FIG. 4. Detailed balance calculations of the dark currents for an ordinary cell and an IBSC.

in Fig. 3 with a dashed line leading to an efficiency of $42.1 \%$ at 1000 suns. For the rest of the cases, the efficiencies are: $36.1 \%$ for the single gap cell (assuming a WL restricted band gap), $40.0 \%$ for the four isolated level IBSC (with four independent QFLs), and $46.9 \%$ for the single level IBSC. A performance reduction is always observed for the multiple QFL case that is, however, smaller when the levels are thermally connected leading to a single QFL.

The dark current density versus voltage plots for the single gap cell, and for the single and multiple level IBSCs are presented in Fig. 4. The most important conclusion to draw is that, at low and high voltages, the dark current is proportional to $\exp (q V / k T)$ for all the plots.

In Fig. 5, the experimental $J_{\mathrm{L}}-V_{\mathrm{OC}}$ plots are presented at concentrations up to 1000 suns for the QD-IBSC (sample A 1681) and a GaAs single junction cell (sample A2191). The $J_{\mathrm{L}}-V_{O C}$ plots appear superimposed on the dark $J-V$ ones (not shown in this letter) except at high currents, where an ohmic voltage drop appears. This is a common behavior for many SCs and is also expected theoretically from the principle of superposition for a single current generator/diode set. However, in the IBSC context, this principle cannot be strictly satisfied by the equivalent circuit (Fig. 2) although the deviations are minimal in practice.

We have fitted the experimental behavior of the QDIBSC with the ideal one by applying constant correction factors (for simplicity) to every photogenerated current and saturation dark current. Furthermore, we have added an $\exp (q V / 2 k T)$ term to the CB-VB diode to account for extra nonradiative recombination. The best fit (Fig. 5) occurs when a single QFL IBSC is considered. It conesponds to $J_{\mathrm{CV}}=J_{0 \mathrm{CV}} \exp (e V / k T)$ with $J_{0 \mathrm{CV}} 27000$ times the van

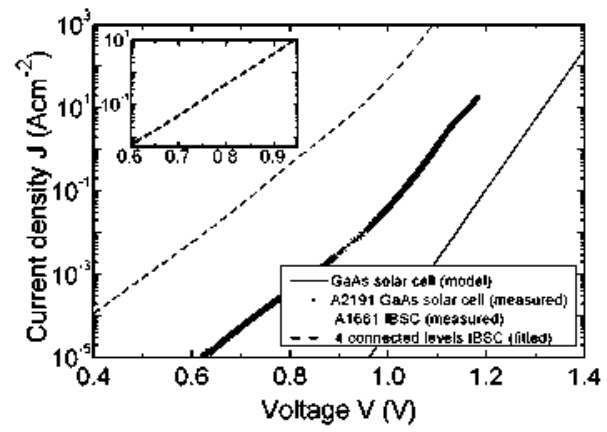

FIG. 5. Experimental $J_{\mathrm{L}}-V_{O C}$ of A2191. a single junction GaAs SC and Al681. a QD-IBSC. The fitted curve of a two exponential characteristic with the single QFL forr-IB levels is also presented. 
Roosbroeck-Shockley term to which we have added $2.8 \sqrt{J_{C V}}$ to account for the $\exp (\mathrm{eV} / 2 k T)$ term. The sum of the van Roosbroeck-Shockley terms $\Sigma_{j} J_{0 I \mathrm{~V}}$ (the term to use when all the nodes $I i$ are interconnected) is multiplied by a factor of 12 ; the sum of the van Roosbroeck-Shockley terms $\sum_{\mathrm{r}} J_{0 I \mathrm{C}}$ is multiplied by a factor of 2 .

All these fits have a reasonable physical explanation. The large factor of 27000 accounts for a considerable deterioration of the cell when the QDs are added, not associated specifically with the IB region but with the whole cell volume which is much larger. This deterioration also involves an $\exp \left(q^{V / 2 k T)}\right.$ term. The factor of 12 for IB-VB recombination represents some nonradiative mechanisms that are relatively weak. The factor of 2 associated with CB-IB recombination probably reveals a reduction in the radiative recombination due low absorbance (that equals the emittance). This is found in other experiments, ${ }^{9}$ that would lead, by itself, to a lower factor $<1$, compensated by some nonradiative recombination that is also expected to be present. However, the final impact of this nonradiative recombination is not large because the maximum radiative saturation current (obtained when the emittance is one) is so strong $\left(0.073 \mathrm{~A} \mathrm{~cm}^{-2}\right)$ that nonradiative recombination does not become much larger.

In summary, the presence of multiple levels to form IBs reduces the efficiency limit of the IBSC derived from detailed balance calculations, the worst case being when all the IB levels have different QFLs.

$J_{\mathrm{L}}-V_{\text {OC }}$ measurements up to 1000 suns show that the use of QDs in the IBSC results in a strong two-exponential char- acteristic, over four orders of magnitude above the detailed balance limit, that is thought to be associated with the whole cell volume and not restricted to the IB region. In contrast, the nonradiative recombination associated with the IB levels is not large and in the case of the CB-IB recombination is in the range of the ideal radiative recombination (that is rather high for the low band gap).

This work has been supported by the IBPOWER Grant No. 211640 of the European Commission, by the GENESIS FV Grant No. CSD2006-0004 of the Spanish program CONSOLIDER, and by the NUMANCIA Grant No. S-0505/ENE/ 0310 of the Comunidad de Madrid.

'A. Luque and A. Martí. Phys. Rev. Lett. 78, 5014 (1997).

${ }^{2}$ A. Luque, A. Martí, C. Stanley, N. López, L. Cuadra, D. Zhou, and A. Mc-Kee, J. Appl. Phys. 96, 903 (2004).

${ }^{3}$ S. M. Hubbard, C. D. Cress, C. G. Bailey, R. P. Raffaelle, S. G. Bailey, and D. M. Wilt, Appl. Phys. Lett. 92, 123512 (2008).

${ }^{4}$ S. A. Blokhin, A. V. Sakharov, A. M. Nadtochy, A. S. Panysov, M. V. Maximov, N. N. Ledentsov, A. R. Kovsh, S. S. Mikhrin, V. M. Lantratov. S. A. Mintairov, N. A. Kaluzhniy, and M. Z. Shvarts, Seniconductors 43. $514(2009)$.

${ }^{5}$ A. Luque and A. Martí. Adv. Mater. 22, 160 (2010).

${ }^{6}$ E. Cánovas. A. Martí. N. López, E. Antolín, P. G. Linares, C. D. Farmer. C. R. Stanley, and A. Luque. Thin Solid Filns 516.6943 (2008).

${ }^{7}$ V. Popescu, G. Bester, M. C. Hanna, A. G. Norman, and A. Zunger. Phys. Rev. B 78. 205321 (2008).

${ }^{8}$ W. van Roosbroeck and W. Shockley. Phys. Rev. 94. 1558 (1954).

${ }^{9}$ A. Martí. E. Antolín. C. R. Stanley. C. D. Farmer, N. López. P. Diaz. E. Cánovas, P. G. Linares. and A. Luque. Phys. Rev. Lett. 97. 247701 (2006) 